\title{
Le temps des coachs?
}

Trajectoires typiques d'une figure du « nouvel esprit du capitalisme »

Time for coaches? Typical careers of a figure of the "new spirit of capitalism"

\section{Scarlett Salman}

\section{OpenEdition}

\section{Journals}

Édition électronique

URL : http://journals.openedition.org/travailemploi/6713

DOI : 10.4000/travailemploi.6713

ISSN : 1775-416X

Éditeur

DARES - Ministère du Travail

Édition imprimée

Date de publication : 1 juillet 2015

Pagination : 59-73

ISSN : 0224-4365

Référence électronique

Scarlett Salman, «Le temps des coachs ? », Travail et Emploi [En ligne], 143 | juillet-septembre 2015 mis en ligne le 01 juillet 2017, consulté le 01 mai 2019. URL : http://journals.openedition.org/ travailemploi/6713; DOI : 10.4000/travailemploi.6713 


\title{
Le temps des coachs? Trajectoires typiques d'une figure du " nouvel esprit du capitalisme "
}

\author{
Scarlett Salman $\left.{ }^{*}\right)$
}

\begin{abstract}
Développé aux États-Unis dans les années 1980-1990, rapidement introduit en France, le coaching en entreprise suscite la constitution d'un segment professionnel en plein essor dans l'espace du conseil des années 2000. Dégager les conditions sociales et les raisons qui conduisent un ensemble d'acteurs à se tourner vers cette activité permet de montrer comment se consolide ce nouveau segment professionnel, au-delà de l'action des premiers militants engagés dans sa reconnaissance. Notre enquête dégage deux principales voies d'entrée pour devenir coach: la première comprend d'anciens consultants, formateurs ou psychologues à la trajectoire professionnelle "buissonnante»; la seconde est le fait de cadres d'entreprise reconvertis après une rupture dans une carrière salariée auparavant linéaire. Le cas du coaching renseigne ainsi sur les affinités électives entre le "nouvel esprit du capitalisme» (BOLTANSKI, CHIAPELLO, 1999) et des trajectoires professionnelles qui soulignent l'attractivité contrainte des formes hybrides d'emploi.
\end{abstract}

Encensés ou décriés, le «nouvel esprit du capitalisme» (Boltanski, Chiapello, 1999) ou les discours de «la nouvelle entreprise» (SOCIÉTÉS CONTEMPORAINES, 2013) désignent l'évolution de la littérature managériale dans les années 1990 et les politiques managériales des grandes entreprises dans les années 2000 (dont les maîtres mots sont alors autonomie, flexibilité, projet, évaluation et individualisation des compétences, adaptabilité et employabilité, etc.). Si les consultants, qui portent ces représentations et ces pratiques dans les entreprises, et jouent donc un rôle important dans la structuration du capitalisme, sont de plus en plus étudiés (HENRY, 1992, 2012; BERREBIHoffmann, 2002; Kipping, Engwall, 2002; Villette, 2003 ; GIRAUD, 2007; THINE et al., 2013), le projecteur est généralement mis sur les consultants des cabinets de conseil, qui sont quantitativement dominants dans le conseil en management, en informatique, en audit et en expertise comptable. Ce faisant, ces études laissent dans l'ombre une partie singulière de l'espace du conseil, composée de consultants indépendants ou de toutes petites structures de deux à trois personnes. Or, une activité emblématique du «nouvel esprit du capitalisme», le coaching, est exercée par des consultants qui se trouvent en dehors des organisations du conseil et qui le revendiquent. Par les formes hybrides d'emploi qu'ils occupent ${ }^{(1)}$ - les «habits neufs de l'indépendance» (Menger,

(*) Laboratoire interdisciplinaire sciences, innovations, sociétés (Lisis), université Paris-Est Marne-la-Vallée; Scarlett. Salman@u-pem.fr

(1) Gérant ou assimilé salarié d'une société à responsabilité limitée (SARL), dirigeant de cabinet, salarié «porté» par une société de portage salarial, entreprise unipersonnelle à responsabilité limitée (EURL), etc. Seule une minorité est salariée de grandes entreprises (coachs internes) ou de cabinets de conseil.
2002) - les coachs illustrent la figure, valorisée dans cette idéologie, de l'entrepreneur de soi-même, du «professionnel autonome» (REYNAUD, 2007), qui accompagne la remise en cause du salariat pour certains métiers de la connaissance (BARLEY, KUNDA, 2004).

Développé aux États-Unis au tournant des années 1980-1990, le coaching est presque aussitôt introduit en France par des consultants qui ont la particularité d'exercer également comme psychothérapeutes (SALMAN, 2003). Il est plus largement issu d'une hybridation entre la psychosociologie du travail qui a façonné les champs du management et de la formation (Boltanski, 1981; TANGuY, 2001) d'une part, et la psychothérapie du développement personnel (STEVENS, 2011), face au relatif échec de cette dernière à se faire une place dans un champ de la santé mentale dominé par des acteurs institutionnels (psychiatres, psychanalystes et psychologues) (Champion, 2008), d'autre part. Consistant en une dizaine d'entretiens individuels, confidentiels et réguliers entre un consultant coach et un cadre, la prestation qu'ils délivrent est définie comme «l'accompagnement de personnes pour le développement de leurs potentiels et de leurs savoir-faire, dans le cadre d'objectifs professionnels ${ }^{(2)} \gg$. Emblématique

(2) Selon la Société française de coaching (SF Coach), la plus ancienne association professionnelle de coachs en France, qui a été fondée à l'initiative d'une dizaine de consultants psychothérapeutes en 1997, quatre ans avant la création de l'antenne française de l'association américaine International Coach Federation (ICF). La SF Coach représente encore aujourd'hui symboliquement la principale association française de coachs. Sans être devenue l'unique référence des professionnels, la définition qu'elle donne du coaching s'est néanmoins progressivement imposée comme représentative de l'activité (SALMAN, 2013). 
des politiques néomanagériales qui visent à transformer les cadres en «leaders» porteurs d'une «vision» et à favoriser l'épanouissement personnel au nom de la performance, le coaching a été mis en place dans les grandes entreprises au cours des années 2000 en France, contribuant à l'individualisation et à la psychologisation des problèmes de travail et de carrière des cadres (SALMAN, 2008). Le recours au coaching a donné naissance à un nouveau segment professionnel rapidement en plein essor, sous l'impulsion de militants engagés, dès la deuxième moitié des années 1990, dans la lutte pour la reconnaissance d'une «juridiction»spécifique (Аввотт, 1988) au coaching, entre conseil et psychothérapie. L' «offre» de coaching, qui se manifeste par la création d'associations professionnelles, de formations privées ou universitaires, et par l'arrivée sur le marché du conseil de praticiens proposant cette activité en tant que coachs, est en effet passée d'environ 250 personnes dans la deuxième moitié des années 1990 à près de 4000 à la fin des années $2000^{(3)}$

Le coaching est donc rapidement apparu comme un débouché professionnel à une multitude d'acteurs. Que révèle cet engouement ? Dégager les conditions sociales et les raisons qui conduisent à se tourner vers cette activité permet d'abord de montrer comment se consolide un nouveau segment professionnel, au-delà de l'action des militants engagés dans sa reconnaissance. Plus encore, l'analyse du coaching par le biais de ceux qui l'exercent, dans la mesure où ils apparaissent emblématiques de l'éthos du «nouvel esprit du capitalisme», fournit une chance sociologique de pouvoir saisir ce qui motive les croyances en ce «nouvel esprit» : quels types d'acteurs y sont réceptifs? Pour quelles raisons et dans quelles configurations ? Quelles conditions sociales, quelles transformations du travail et de l'emploi les ont conduits à adhérer à ces représentations typiques de la «cité par projets ${ }^{(4)}$ » (voir encadré 1) et à contribuer à leur diffusion ?

Comme Hélène STEVens (2011), on peut regretter que la transition entre «l'esprit du capitalisme» des années 1960 et celui des années 1990 ne soit pas

(3) Selon nos estimations croisant le nombre de personnes formées au coaching avec le nombre d'adhérents aux principales associations (SALMAN, 2013).

(4) La notion de «cité par projets» (Boltanski, Chiapello, 1999) a été forgée à partir d'une étude systématique d'un corpus de textes de management des années 1990. Les termes de réseau, de connexion, de flexibilité, d'activité, de projet mais aussi de créativité et de singularité également mises en avant par la «cité inspirée», y occupent une place centrale. Le coach y est valorisé comme celui qui «éveille» les esprits. Nous ne considérons pas que ces discours correspondent à des pratiques effectives dans les entreprises; nous montrons au contraire, par ailleurs (SALMAN, 2014), que la pratique du coaching va plutôt à l'encontre des principes professés par le néomanagement. En revanche, le discours managérial a sensiblement changé dans les années 1990. C'est précisément à une étude du groupe social qui tient ce nouveau discours que cet article souhaite contribuer. suffisamment documentée, rendant difficilement compréhensible le processus de récupération de la «critique artiste» par le capitalisme mis en évidence par Boltanski et Chiapello (1999). Une partie de la réponse nous semble se trouver dans l'analyse des investissements d'un ensemble d'acteurs dans une activité emblématique de cette tendance, en complément de l'étude des transformations économiques et productives qui ont contribué à façonner les conditions favorables à de telles trajectoires. Parce qu'il repose sur les intérêts diversifiés d'acteurs hétérogènes, le groupe professionnel des coachs constitue un objet propice pour éclairer les ressorts de l'adhésion au «nouvel esprit du capitalisme». Une telle étude permet de sortir d'une vision univoque des entrepreneurs du néomanagement, en soulignant au contraire les significations diverses dont peut être investi ce discours en fonction des parcours variés qui coexistent et entrent en concurrence au sein du groupe des coachs.

L'examen des trajectoires biographiques de celles et ceux qui sont devenus coachs au tournant des années 1990-2000, en général après leurs 45 ans, dégage en effet deux principales voies d'entrée dans le coaching : des consultants et des formateurs pour qui le coaching représente, dans une trajectoire buissonnante ${ }^{(5)}$, une opportunité professionnelle qui devient une ligne directrice autour de laquelle se réorganisent les différentes activités qu'ils exercent; d'anciens cadres salariés pour qui le coaching est une reconversion professionnelle après une rupture (licenciement, accident, etc.) dans une carrière auparavant relativement linéaire. L'article se structure autour de ces deux idéaux-types, qui épousent la dynamique de construction de l'espace professionnel par deux moments de la diffusion du coaching. Tandis que le premier profil rassemble plutôt des promoteurs de l'institutionnalisation de cette activité, les cadres qui relèvent du second se sont tournés vers le coaching à leur suite : de clients, ils sont devenus praticiens et parfois instigateurs de la mise en place du coaching au sein de leur entreprise. Outre l'attractivité de formes hybrides d'emploi auprès d'acteurs relevant des franges du conseil, notre article contribue ainsi à montrer tout ce que les reconversions professionnelles «volontaires » (NÉGroni, 2007; Denave, 2015) doivent aux transformations de l'emploi et des carrières des cadres des vingt dernières années, ainsi qu'aux dispositifs d'accompagnement des transitions professionnelles soutenus par les pouvoirs publics, derrière la rhétorique professionnelle enchantée de la vocation et de l'indépendance tenue par les «(re)convertis».

(5) «Dont les divisions sont nombreuses dès la base et les branches irrégulières» (dictionnaire Robert). Nous désignons ici tant la multiplicité des activités exercées que celle des statuts dans l'emploi des coachs. 


\section{Encadré 1 \\ Portrait du coach en valeurs}

Bien qu'elles soient diversement appropriées selon les trajectoires biographiques et les propriétés sociales, les représentations collectivement construites par les coachs permettent d'identifier "un discours de coach" comportant plusieurs pôles.

Un premier pôle valorise l' "entreprise de soi ", la promotion du sujet, de l'individu, qui va de pair avec une critique, pêle-mêle, des institutions, des organisations, des bureaucraties, de l'autorité, des relations asymétriques, du salariat. II rejoint les appels managériaux faits aux salariés d'être «entrepreneurs » d'eux-mêmes (STEVENS, 2012). Imprégnée de la "critique artiste(1)» qui s'exprime lors de Mai 1968, dans le sillage de la contre-culture, l'“entreprise de soi » est d'abord portée par les introducteurs du coaching en France et vite adoptée par les "convertis". Face aux institutions dénoncées comme inhibitrices et porteuses d'illusions, est affirmée la croyance en un "soi (2)», potentiel infini à développer, selon la formule «deviens qui tu es». C'est au nom de soi que se fait la bifurcation vers le coaching, perçue comme une émancipation. L'action, les «winners», les "guerriers», la multi-activité sont valorisés, au contraire de la passivité et plus encore de la plainte, objet de stigmatisations explicites.

Un deuxième pôle se rattache au “connexionnisme (3)». Les coachs à la trajectoire éclectique sont ceux qui le revendiquent le plus. L'accent est mis sur un «sens de la débrouille», une intelligence pratique, une créativité, qui sont censés davantage devoir au "savoir-être" et aux relations sociales, vues comme autant de connexions, qu'aux qualifications. C'est le discours de celui qui est là au bon moment, qui réussit par les rencontres, par son charisme et non par ses diplômes. Lorsqu'ils se présentent, les coachs utilisent les qualificatifs de "chanceux", "curieux», "audacieux», "brillant», etc. Les échecs sont relus comme autant de détours fructueux. Est valorisé un don-juanisme professionnel : il s'agit de voler d'invention en invention, et de ne pas se laisser enfermer par une activité définitive, afin de montrer qu'on est toujours «jeune». La diversité des activités et des mondes sociaux traversés au cours de sa trajectoire fait émerger la figure du «marginal-sécant ${ }^{(4)}$ », à l'intersection de plusieurs mondes, valorisée dans cette perspective connexionniste.

Enfin, un troisième pôle exprime l'idée d'humanisme économique. Présenté avec une dimension militante - "Faire évoluer un homme dans l'entreprise, c'est déjà participer au changement de la société tout entière (5)", il est au cœur des discours de la vocation : il consiste à aider les autres, en rattachant le coaching à la "relation d'aide ", à «l'accompagnement ", à la «position basse » du coach contre «la position haute de l'expert ». Humaniser l'entreprise renvoie aussi à l'affirmation du rôle des sciences humaines dans la société, ce qui permet de faire du coaching, si ce n'est une discipline, au moins une pratique réflexive, et pas seulement un «business». Ces représentations inscrivent le coaching dans la filiation du courant d'humanisme économique de Jean Coutrot des années 1930, qui visait à introduire les sciences humaines dans les entreprises (HENRY, 2009), et dans le projet de la psychologie sociale américaine de Kurt Lewin de rendre les organisations industrielles plus démocratiques, forgé aux États-Unis dans les années 1930 et relayé en France dans les années 1950 via les "missions de productivité" du plan Marshall (BoltANSKI, 1981). Ce discours est en partie sous-tendu par des valeurs religieuses, la dimension spirituelle du coaching étant affirmée au premier chef par Vincent Lenhardt, consultant catholique qui a le plus contribué à l'introduction du coaching en France dès la fin des années 1980. Également porté par des acteurs formés à la psychologie clinique et à ses valeurs humanistes, il s'appuie enfin sur le parcours heurté d'anciens cadres et le processus proche du «transfert» qui les amène à vouloir aider comme eux ont été aidés. Bien que ce pôle soit proche de la figure du soin, du care, traditionnellement associé au genre féminin, son genre est néanmoins ambivalent, tant le terme de coach y est par ailleurs associé à des valeurs viriles de performance voire de compétition. Les coachs distinguent ainsi l'humanisme et «l'humanitaire», dénigré au nom du principe de responsabilité individuelle. L'un des promoteurs de l'institutionnalisation du coaching en France, résume ainsi, à propos

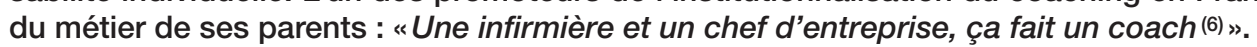

(1) À côté de la «critique sociale» qui dénonce les inégalités et l'égoïsme, la notion de «critique artiste» (ChIAPELLO, 1998) a été construite pour désigner les demandes d'authenticité et de liberté, qui s'enracinent dans l'invention d'un mode de vie bohème au milieu du XIXe siècle et dans la critique de la bourgeoisie par la revendication de la figure de l'artiste. Cette critique trouve une expression plus contemporaine dans la contestation portée par le mouvement de Mai 1968.

(2) L'italique indique qu'il s'agit de notions utilisées par les acteurs, à la différence des notions académiques, également indiquées entre guillemets mais sans italique.

(3) Emprunté aux sciences cognitives, le concept de «connexionnisme» est utilisé par Luc BolTANSKI et Ève CHIAPELLO (1999) pour désigner le développement du travail en réseau et de l'entreprise en réseau, et, au-delà, pour affirmer l'importance que cette configuration a prise dans la structuration de la vie sociale. Pour ces auteurs, l'activité par excellence dans la "cité par projets» consiste à s'insérer dans des réseaux. De nouveau, en utilisant cette notion, nous n'affirmons pas qu'elle désigne des pratiques effectives mais seulement des représentations portées par un certain type d'acteurs, dont les coachs font partie.

(4) Cette figure, définie par Michel Crozier et Erhard Friedberg (1977, p. 73) comme désignant «l'acteur qui est partie prenante dans plusieurs systèmes d'action en relation les uns avec les autres, et qui peut, de ce fait, jouer le rôle indispensable d'intermédiaire et d'interprète entre des logiques d'action différentes, voire contradictoires », est parfois explicitement revendiquée par les coachs. On en trouve un exemple chez Jean-Louis SENTiN (2010). Généralement, la notion est utilisée par les coachs pour valoriser leur position intermédiaire entre le champ «psy» et celui de l'organisation, et se distinguer à la fois des «psys» et des acteurs des ressources humaines et du management.

(5) Page de présentation d'une titulaire de la SF Coach.

(6) Entretiens réalisés les 8 et 20 mai 2007. 
Notons que les trois pôles de représentations (voir encadré 1) sont portés par l'ensemble du groupe des coachs; néanmoins, le premier profil de coachs (des anciens consultants et formateurs) revendique plus souvent le «connexionnisme» tandis que le second profil (des cadres [re]convertis) insiste davantage sur l'humanisme économique, les deux profils tenant par ailleurs fortement le discours de «l'entreprise de soi», bien que pour des raisons différentes qui sont présentées dans la suite de l'article. Cette étude s'appuie sur un ensemble d'entretiens réalisés au cours des années 2000 auprès de coachs en activité (voir encadré 2).

\section{Encadré 2 Matériaux empiriques}

À l'appui de cette recherche, 45 entretiens biographiques ont été menés avec des coachs en entreprise entre 2002 et 2008, principalement en 2006-2007, afin de restituer les conditions objectives et les raisons subjectives de l'orientation professionnelle dans le coaching. Les trois quarts des enquêtés pratiquent le coaching, revendiquent l'exercice du «métier» de coach et sont inscrits dans une association professionnelle. Ce corpus est comparé aux résultats d'une enquête par questionnaire auprès de coachs de la première association professionnelle (116 réponses) en 2002, au début de l'institutionnalisation de cette activité dans les grandes entreprises en France. Ce croisement permet de mieux cerner les caractéristiques des enquêtés. La place importante, dans notre enquête, de coachs occupant des responsabilités dans le groupe professionnel explique la surreprésentation masculine des répondants $(23$ hommes et 22 femmes) alors que le groupe est majoritairement féminin (près des deux tiers en 2010).

\section{Devenir coach, une opportunité pour des consultants et formateurs aux franges du conseil}

Le premier profil de coachs rassemble des consultants et des formateurs qui sont - en comparaison des consultants des grands cabinets de conseil - plus âgés, plus souvent des femmes, moins diplômés et intégrés à l'ordre économique. Si le coaching représente au départ, pour ces acteurs, une corde de plus à leur arc, il leur permet in fine d'étendre leur champ d'action professionnel et de gagner une position plus élevée socialement, économiquement ou symboliquement. Ce premier profil, que l'on peut qualifier d'«opportuniste», a deux ramifications, que nous présentons plus en détail dans les pages qui suivent. Une partie de ces coachs a principalement exercé leur activité antérieure comme indépendants, dans une trajectoire éclectique faite d'emplois nombreux et de séquences d'alternance entre salariat et indépendance, voire de chômage et d'inactivité. D'autres, consultants salariés, ont au nom du coaching quitté leur cabinet de conseil et orienté leur activité vers une pratique proche de la psychothérapie.

\section{Valoriser le parcours éclectique de consultants indépendants}

Parmi nos enquêtés, les consultants et formateurs qui travaillaient comme indépendants avant de devenir coachs ont une trajectoire éclectique, d'abord par la diversité de leurs activités professionnelles au sein d'une famille de métiers relativement limitée, à savoir celle des métiers de service dans le domaine culturel au sens large : l'enseignement, les médias, la formation en ressources humaines, les associations socioculturelles. Ils ont souvent exercé avec un statut non salarié, comme indépendant pour le conseil et la formation, comme vacataire voire bénévole pour les autres activités. Leurs trajectoires sont faites d'une succession de phases d'emploi, de chômage et d'inactivité, et de plusieurs reprises d'études. Le coaching représente alors une opportunité professionnelle attractive car il est peu réglementé, a priori ouvert à tous et susceptible de donner de la valeur à un parcours non linéaire. Bien qu'il s'agisse d'un parcours d'ascension sociale, peu fréquent au sein du groupe professionnel des coachs ${ }^{(6)}$, le cas de Jérôme (voir encadré 3) illustre deux traits saillants de ce profil : un rapport contrarié à l'école et l'orientation vers un métier relationnel peu réglementé. La valorisation des carrières non linéaires, revendiquées comme atypiques, et des «relations» au détriment des qualifications est largement partagée par les coachs.

\section{Un rapport contrarié aux études et à l'enseignement}

Parmi les nombreuses activités qui parsèment les trajectoires éclectiques de ces acteurs, l'enseignement occupe une place proéminente, surtout en début de carrière. Il est cependant toujours présenté comme un choix par défaut. L'enseignement a par exemple constitué, pour plusieurs femmes, une occupation professionnelle sur laquelle elles se sont rabattues après l'arrêt ou l'échec de leur formation universitaire initiale, arrêt qu'elles imputent en partie aux naissances rapprochées de leurs enfants dont elles se sont occupées, suivant ainsi le modèle familial traditionnel. Pour certains hommes issus de classes populaires, comme Jérôme, les vacations d'enseignement ont représenté un travail alimentaire. L'amertume de ces acteurs est à relier aux positions précaires qu'ils ont ainsi occupées, souvent comme vacataires dans des zones défavorisées. Ces conditions d'emploi, choisies en raison

(6) C'est parmi les coachs au parcours éclectique que se trouvent la plupart des coachs en ascension sociale. Toutefois, ils côtoient également des acteurs de milieux sociaux plus favorisés en lutte contre le déclassement social. 


\section{Encadré 3 \\ Jérôme, ou la revanche par le coaching}

L'un des rares enfants d'ouvrier parmi les coachs que nous avons rencontrés, Jérôme, né dans les années 1950 , se lance après son baccalauréat dans des vacations à l'école primaire, dont il garde de mauvais souvenirs, souffrant de son statut de "suppléant éventuel». II débute des études universitaires mais change plusieurs fois d'orientation sans aboutir.

La psychologie comme révélation. Outre l'intérêt qu'il trouve à cette discipline, la psychologie lui ouvre de nouveaux horizons professionnels. II quitte ses vacations d'enseignant pour des fonctions de «rééducateur psychopédagogique", puis de psychologue scolaire de l'Éducation nationale. La loi du 25 juillet 1985 réglementant l'usage du titre de psychologue le contraint à reprendre ses études : il obtient une maîtrise de psychologie à 35 ans.

Une deuxième partie de sa carrière s'ouvre alors : l'entrée dans le champ du conseil et de la formation en entreprise. Après avoir appris que sa maîtrise et ses cinq années d'expérience lui permettaient d'acquérir, par équivalence, le titre de psychologue, il démissionne de l'Éducation nationale et se tourne vers le secteur privé. II devient "consultant-formateur freelance", fait des vacations pour des cabinets de conseil, travaille directement pour des entreprises : formations au management, à la gestion des conflits, missions de marketing, etc. II entend parler pour la première fois d'“accompagnement individualisé au changement" au Québec à la fin des années 1980 et participe à une formation émergente, collaborative et peu coûteuse à cette variante locale du coaching.

La création de la première association professionnelle de coaching en France au milieu des années 1990 agit comme un aimant auprès de nombreux consultants multicartes comme Jérôme. Ce dernier cherche à stabiliser sa situation professionnelle et le coaching représente une opportunité professionnelle pour le faire, d'autant qu'il peut se prévaloir de l'antériorité de son expérience québécoise. II devient l'un des cadres de l'association et crée une nouvelle structure de conseil et de formation centrée sur «l'accompagnement».

La valorisation ex post de son parcours par le coaching : y compris dans ce qu'il a de non linéaire et de foisonnant. La thématique de «l'accompagnement» lui permet de relire ses différentes activités sous cet angle et de se démarquer de l'enseignement :

"Je crois que le fil rouge du coaching pour moi, ça a toujours été mon intérêt pour l'accompagnement: accompagner l'autre, l'aider à dépasser une difficulté ou à grandir et je l'ai fait avec des enfants, je l'ai fait avec des adultes, dans l'univers professionnel. »

Reprenant l'opposition entre l'individu et la société (ELIAS, 1991), il entend se mettre au service du développement de l'individu, contre une société vue comme oppressive et se targue d'avoir participé au mouvement de Mai 1968 comme «militant lycéen trotskiste».

La diversité de ses activités professionnelles antérieures, y compris de ses errances universitaires, est présentée comme une richesse, offrant un intérêt professionnel spécifique, ce qui lui permet de prendre sa revanche sur le système scolaire. C'est la position du «marginal-sécant», qui peut se prévaloir de son appartenance à différents mondes sociaux : " J'ai beaucoup flirté toujours aux frontières des systèmes sans vraiment rentrer dedans. 》Cette caractéristique fait de Jérôme un vecteur actif de la diffusion de la psychologie du développement personnel(1) dans les différentes sphères où il a circulé, en particulier l'école et l'entreprise.

(1) Et des critiques faites à l'école, transférées au monde de l'entreprise (LE GofF, 1999).

même de leur flexibilité, sont mal vécues in fine. La critique partagée de l'enseignement à l'Éducation nationale prend appui sur le sentiment d'un choix contraint et sur l'absence de détention des diplômes adéquats pour être titulaire.

Les coachs au parcours éclectique sont en effet moins diplômés que le groupe des coachs dans son ensemble. Titulaires par exemple d'une licence en sciences humaines, ils l'ont obtenue lors de leur formation initiale mais au terme d'une scolarité perturbée par plusieurs orientations. Autre indice de ce rapport contrarié aux diplômes : l'importance de la reprise d'études, qui concerne une très large majorité des coachs. Chez les coachs au parcours éclectique, celle-ci est marquée par la volonté de compenser des diplômes initiaux jugés inadaptés. Le rapport aux diplômes est néanmoins ambivalent pour eux : ils disent n'accorder que peu de valeur aux qualifications en comparaison des compétences et du «savoir-être» des personnes, mais ils mettent en évidence des titres qu'ils n'ont parfois pas ou qu'ils enjolivent. Cette ambivalence semble confirmer ce que ce discours critique doit à leur parcours scolaire non linéaire. On retrouve chez eux des caractéristiques proches des formateurs d'adultes, lesquels avaient déjà instauré la formation professionnelle en s'appuyant sur la critique de l'enseignement scolaire mis en place par l'Éducation nationale (Prost, 2008). Sans être aussi proches des «frontières de l'école» que les animateurs détenteurs du Bafa (7), les coachs sont, eux aussi, porteurs d'une «pédagogie anti-scolaire» (PINTO, 2010).

(7) Brevet d'aptitude aux fonctions d'animateur. 


\section{L'attrait pour une nouvelle voie peu réglementée et relationnelle : la figure du " connexionnisme"}

Les coachs au parcours éclectique ont trouvé des issues professionnelles en reprenant des études de psychologie, de psychosociologie, de sciences de l'éducation ou en suivant des formations en développement personnel, certes moins académiques mais alors présentées comme un vrai choix, au contraire des cursus universitaires. Tous ceux que nous avons rencontrés ont eu recours à une psychothérapie, et certains relient leur intérêt pour la psychologie à une révélation survenue dans un contexte personnel éprouvant.

Les trajectoires de ces coachs se caractérisent par leur orientation vers des métiers «à classement flou », définis comme des «métiers à grande dispersion et peu professionnalisés, et dans les secteurs les plus nouveaux de la production culturelle [...], où les postes et les carrières n'ont pas encore acquis la rigidité des vieilles professions bureaucratiques et où le recrutement se fait encore, le plus souvent, par cooptation, c'est-à-dire sur la base des "relations" et des affinités d'habitus, plutôt qu'au nom des titres scolaires» (Bourdieu, 1979, p. 168). Ces trajectoires concernent par exemple des femmes mariées à des cadres d'entreprise, qu'elles ont suivis dans leur évolution de carrière, et ont poursuivi avec le coaching un accompagnement qu'elles exerçaient, dans l'ombre, auprès de leur mari. Elles concernent aussi certains hommes peu diplômés et en ascension sociale. Tous s'étaient tournés vers des activités requérant à l'époque peu de qualifications, et s'obtenant plutôt par relation : vacations dans le journalisme, la radio, l'animation commerciale, la formation, etc. Un ancien animateur commercial puis animateur radio insiste sur l'importance des «rencontres» dans sa carrière et rassemble les métiers qu'il a exercés sous la bannière de la «communication». Ces acteurs tentent de valoriser leur parcours en mettant en avant un "sens de la débrouille», une intelligence des situations, qui s'apparente à la mètis des Grecs, intelligence fondée davantage sur la ruse que sur la raison (Detienne, Vernant, 1974). Ils mobilisent ces « compétences » comme un argument qui attesterait de leurs qualités de coach, jetant un voile d'ignorance sur les conditions qui leur ont permis d'atteindre leurs positions socioprofessionnelles (capital social, économique, culturel, acquis par leur famille ou leur conjoint, en particulier pour les femmes). Ils se présentent comme le type même de l'individu «connexionniste» qui sait constituer et utiliser des réseaux de relations, figure du médiateur de "la cité par projets», qui soutient normativement le «nouvel esprit du capitalisme» (Boltanski, Chiapello, 1999).

\section{Revendiquer son caractère atypique}

Liée à la valorisation du «connexionnisme» (voir encadré 1), la présentation du parcours professionnel comme "atypique» est la deuxième forme de justification massivement adoptée par ces coachs pour réinterpréter positivement leur itinéraire :

«Alors, on dira qu'il [mon parcours] est assez atypique dans la mesure où il n'est pas linéaire. Et-c'est un mot dont vous avez sûrement entendu parler - il n'est pas sur des rails. On ne m'a pas dit un jour, ou je ne me suis pas dit un jour: "Je ferai telle carrière. Et je ferai ça toute ma vie”.»

(Jean-François, coach indépendant, ancien animateur commercial, 50 ans, entretien réalisé le 8 octobre 2006.)

De fait, les trajectoires non linéaires de ces coachs sont atypiques au sein de la population active en France, où le schéma traditionnel des carrières professionnelles stables dans une même organisation perdure, en dépit de tous les discours vantant le «nomadisme» et la mobilité (DE LARQUIER, RÉmillon, 2008). Ce caractère atypique s'explique d'abord par les domaines professionnels dont ils sont issus, la formation et la communication, marqués par une plus forte instabilité professionnelle que la moyenne, due à l'importance structurelle des contrats courts et du chômage (Simmonet, Ulrich, 2009). Pour ces consultants indépendants pluriactifs, le coaching s'inscrit, dans leur trajectoire buissonnante, comme l'opportunité d'unifier leurs activités sous un titre valorisé dans le marché du conseil. L'oscillation entre des phases d'emploi où alternent indépendance et salariat, voire inactivité, est en partie liée, pour les femmes, à un modèle traditionnel de répartition sexuée des rôles au sein du couple. Parce qu'il s'exerce majoritairement sous des statuts non salariés, le coaching représente pour elles l'opportunité d'un retour vers l'emploi.

Au-delà des trajectoires individuelles, la figure du coach comme personnalité atypique empruntant des chemins de traverse fait l'objet d'une construction collective de la part du groupe professionnel. Le terme d'«atypique» a l'intérêt de montrer sa singularité - argument de vente du coach - dans un monde critiqué pour ses normes et sa standardisation, au nom de l'authenticité et de la liberté. Plus encore, l'expérience personnelle, même hasardeuse, est vue comme un gage de professionnalité dans l'ordre de valeurs du «nouvel esprit du capitalisme ${ }^{(8)} \gg$. Cette représentation de soi permet de retourner le stigmate associé à des diplômes

(8) «Un capitalisme qui s’incorpore des justifications connexionnistes accepte, contrairement à l'ancienne société bourgeoise, ceux qui doivent à un parcours de vie relativement erratique, au moins dans leur jeunesse, un capital d'expériences et une connaissance de plusieurs mondes leur conférant une adaptabilité importante.» (BOLTANSKI, ChIAPELLO, 1999, p. 176.) 
inadéquats en faisant du caractère buissonnant de son parcours sa principale richesse, voire une garantie de qualité professionnelle, à l'instar de cette coach qui n'est pas diplômée de psychologie universitaire :

"C'est l'inconvénient d'être toujours un petit peu en marge. Finalement, ça vient du fait que je n'ai pas choisi les bonnes études. Au début, j'aurais dû faire des études de psycho et puis continuer après. [...] Mais par certains côtés, je me dis, le bon côté des choses c'est que ça m'a obligée finalement à trouver mon propre chemin et à ne pas être entièrement "câblée" d'une certaine manière. "

(Pierrette, 65 ans, entretien réalisé le 9 mai 2007.)

Le coaching permet ainsi de donner un sens et une unité à une trajectoire éclectique et d'offrir une activité professionnelle à des individus retirés du marché du travail ou qui ne détiennent pas les diplômes correspondant à leurs aspirations.

\section{Le coaching comme opportunité de passage du conseil à la psychothérapie}

Au sein du premier grand idéal-type de coachs, pour qui le coaching représente l'opportunité de donner une cohérence à une trajectoire buissonnante, un deuxième sous-ensemble peut être identifié : il est composé d'anciens consultants salariés formés à la psychologie au cours de leurs études initiales - il s'agit surtout de femmes. Bien que proportionnellement moins nombreux que les coachs au parcours éclectique qui viennent d'être présentés, ils jouent un rôle important dans la dynamique d'autonomisation du coaching comme segment professionnel parce qu'ils sont souvent issus de cabinets de conseil en ressources humaines. En se prévalant de leur bonne connaissance du «conseil», ils revendiquent la singularité du coaching, tant dans les pratiques que dans ses modalités d'exercice. Ils incarnent le double caractère de filiation et de rupture du coaching à l'égard du conseil en ressources humaines.

\section{Encadré 4 \\ Brigitte, ou comment le coaching permet à une psychologue contrainte de travailler en entreprise de revenir à ses premières amours}

Titulaire d'un DESS(1) de psychopathologie dans les années 1970, Brigitte a "la chance» d'être recrutée comme psychologue dans un hôpital psychiatrique. Elle y découvre les thérapies familiales systémiques, en parallèle d'une psychanalyse commencée par désir de devenir psychothérapeute.

Une rupture de carrière pour des raisons familiales. Cette rupture confronte Brigitte à la rareté des débouchés pour les psychologues cliniciens. Elle quitte en effet son poste au milieu des années 1980 pour suivre son mari, cadre dirigeant, à Paris. Elle décrit la situation difficile d'une psychologue qui souhaite devenir psychothérapeute et se heurte au peu d'emplois existant alors dans le secteur institutionnel comme dans le secteur libéral. La reconversion apparaît comme une nécessité. C'est par son mari qu'elle retrouve une activité, dans la communication en entreprise.

L'évolution vers le conseil en ressources humaines. Alors que l'entreprise pour laquelle elle travaille traverse une période de restructurations lors de la crise économique de 1993, elle fait partie d'une équipe mandatée pour orchestrer un plan social de grande ampleur. Licenciée à son tour avec le reste de l'équipe, elle participe avec ses collègues à la création d'un cabinet de conseil spécialisé dans l'accompagnement des plans sociaux. Elle découvre les tests d'aptitude et de personnalité, tout en introduisant dans sa pratique des entretiens individuels, dans le cadre des premiers bilans de compétences.

Le retour dans le champ de la psychothérapie à la faveur du coaching. Dans la deuxième moitié des années 1990, elle s'inscrit à la première association française de coachs, le coaching lui apparaissant comme une manière de se rapprocher de la psychothérapie et de quitter le conseil en ressources humaines:

«Je veux retrouver le métier de thérapeute. [...] J'ai besoin de me retrouver avec mes pairs, j'en ai marre du conseil en management... J'ai envie de retrouver les miens. [Les coachs], c'est pas des thérapeutes encore, mais ce sont les miens. "

Le coaching représente une véritable transition entre les deux mondes, ce que confirme l'usage que fait Brigitte du pronom possessif «les miens» pour désigner d'abord ce qu'elle appelle les «thérapeutes» - la suppression du préfixe «psy» renforce la proximité entre coachs et psychothérapeutes, ces derniers ne relevant plus uniquement de la sphère "psy» - puis les coachs. Bien plus, c'est par cette association qu'elle se rapproche d'un psychiatre, spécialiste reconnu des thérapies familiales, devenu coach lui aussi, qui lui propose d'intégrer son réseau de psychothérapeutes. Depuis le début des années 2000, Brigitte quitte progressivement le cabinet de conseil en management pour exercer comme psychothérapeute et coach. Quinze ans plus tôt, elle avait échoué à pénétrer les réseaux de psychothérapie avec son diplôme de psychologue et son expérience à l'hôpital psychiatrique. En revanche, quand elle fait valoir son passage par le conseil en management et son intérêt pour le coaching, elle obtient aisément une place dans un réseau de psychothérapeutes.

(1) Diplôme d'études supérieures spécialisées. 
Leur parcours est marqué par une oscillation entre la psychologie et différentes activités de conseil en ressources humaines (recrutement, gestion des cadres, bilans de compétences, etc.). Le coaching donne a posteriori une cohérence à une trajectoire hybride, qui hésite entre psychologie clinique et conseil en entreprise. La découverte du coaching offre l'opportunité de quitter le conseil et d'exercer une pratique clinique tout en restant dans le monde de l'entreprise. Si ces consultantes et consultants ont quitté des emplois salariés pour embrasser des formes d'emploi relevant de l'indépendance, ce changement de statut s'est fait progressivement, sans rupture pour la plupart, en raison de leur position dans le cycle de vie (ils ont entre 45 et 55 ans) et de la stabilité assurée par les revenus de leur conjoint, souvent cadre dirigeant. Leurs raisons de se tourner vers le coaching peuvent être appréhendées par le parcours de Brigitte (voir encadré 4).

$\mathrm{Ce}$ cas illustre une dynamique qui fait du coaching, pour des consultants ayant une formation de psychologue, une opportunité d'exercer une activité à contenu largement clinique, et de se rapprocher, à plus ou moins long terme, de l'exercice de la psychothérapie. Le parcours de Brigitte est une illustration un peu extrême de cette dynamique, dans la mesure où elle a commencé sa carrière comme psychologue psychothérapeute à l'hôpital et où le coaching lui a permis de retrouver cette activité, avec un statut d'indépendant cependant. Mais il a le mérite de mettre au jour des mécanismes plus implicites dans les autres trajectoires.

\section{Face à un projet professionnel contrarié, l'opportunité du conseil en ressources humaines}

La définition du métier de coach en entreprise semble hantée par la figure du psychologue clinicien, tant elle correspond à l'image qui lui est classiquement associée: une activité clinique fondée sur l'écoute de l'individu dans un but de relation d'aide. Nombreux sont les coachs qui mettent l'accent sur la dimension d' «accompagnement» dans la pratique du coaching, et c'est particulièrement le cas des consultantes qui ont une formation initiale en psychologie. Ces dernières présentent leur intérêt pour la psychologie clinique comme une vocation ancienne, d'autant plus forte qu'elles ont étudié dans des lieux acquis à cette orientation (dans les années 1970, les études de psychosociologie à Nanterre, de psychologie à Censier ou à Vincennes en sont par exemple emblématiques).

Si ces coachs viennent de la psychologie clinique, elles réalisent une grande partie de leur carrière comme consultantes en ressources humaines. Les débouchés en psychologie clinique restent faibles et cantonnés au secteur sanitaire et social, aux emplois moins stables et moins rémunérateurs que ceux des entreprises. Face à cette configuration peu favorable aux diplômés de psychologie, le conseil en ressources humaines est en plein essor dans les années 1980. Leur orientation vers le conseil, perçue dans un premier temps comme une "évasion», est encouragée par un entourage familial souvent proche du monde de l'entreprise.

\section{Critiques croisées du conseil en ressources humaines et du salariat}

Cependant, le conseil a entraîné un certain nombre de déconvenues chez ces coachs. Le recrutement, activité majeure du conseil en ressources humaines, est présenté comme antithétique à l'approche clinique, comme une activité standardisée, qui se fait «à la chaîne », au détriment de la qualité :

«Je détestais le recrutement [...]. Je détestais le nondialogue, je détestais justement l'idée du modèle, c'est-à-dire qu'on a un modèle idéal et on voudrait que la personne rentre dans le modèle. »

(Geneviève, cofondatrice de la première association de coachs, 55 ans, entretien réalisé le 4 mai 2007.)

Cette critique soutient l'opposition entre conseil et coaching, enjeu de la reconnaissance de la spécificité du coaching. Les arguments du «surmesure», de "l'artisanat» et de la maïeutique se rejoignent pour valoriser un dispositif individualisé et personnalisé. C'est au nom de la singularité et de l'authenticité du «sujet», presque érigé en valeur morale, que se fait la critique du conseil, qui vise le recrutement mais aussi les grands cabinets de conseil anglo-saxons :

«Souvent on récupère des déçus des grands cabinets d'organisation, des clients qui sont déçus d'avoir travaillé avec des gros cabinets, comme Mac Kinsey, BCG..., dans lesquels, et je les connais, ils ont trouvé beaucoup d'intelligence et des solutions souvent toutes faites qui ne tiennent absolument pas compte de l'état de l'entreprise à laquelle s'appliquent leurs conseils. »

(Jacques, cofondateur de la première association de coachs, 55 ans, entretien réalisé le 16 mars 2007.)

Cette critique correspond sans doute à la seule stratégie que peuvent adopter des petites structures ou des indépendants sur le marché du conseil en management où les grands cabinets sont dominants. Néanmoins, les coachs revendiquent ce faisant un segment qui leur est propre, d'autant plus que se mêle, à une critique du conseil en recrutement, une offensive contre le travail salarié. Cette critique reprend le thème du «sujet », puisque c'est «au nom de soi» que se font les revendications conduisant à se mettre à son compte :

\footnotetext{
«Je me sentais trop investie dans cette activité qui, comme toutes les activités en cabinet, avait aussi des contraintes en termes de chiffres d'affaires, de rentabilité. [...] J'avais envie de récupérer du temps, [...] du temps pour penser, du temps pour écrire, du temps pour faire un peu différemment les choses. »

(Marie-Claude, coach et psychanalyste indépendante, 50 ans, entretien réalisé le 6 septembre 2007.)
} 
$\mathrm{Si}$ ces consultantes et consultants affichent a posteriori leur souhait de devenir coach pour expliquer leur sortie du salariat, on peut néanmoins se demander s'ils ne cherchaient pas tout autant à quitter leur cabinet de conseil : le coaching a alors représenté une opportunité suffisamment attractive pour pouvoir le faire. La plupart de ces coachs, des femmes nous l'avons dit, ont plus de 45 ans quand elles quittent le cabinet : elles ont moins de contraintes familiales, leurs enfants étant plus âgés; elles ont peu de difficultés financières car elles ont eu des salaires élevés comme consultantes; plusieurs d'entre elles sont mariées à des cadres dirigeants de grandes entreprises et jouissent du patrimoine familial. Elles expriment un sentiment de libération des contraintes liées à la maternité et au travail, et disent rechercher un "équilibre de vie» plutôt qu'une «intensité de travail». Leur critique du conseil se nourrit des insatisfactions ressenties en tant que consultantes. En opposant, d'une part, l'organisation et ses contraintes, le conseil et son caractère standardisé et, d'autre part, l'accent mis par le coaching sur l'individu, le sujet, la singularité, elles parlent également de leur propre sortie de l'organisation, de leur propre recherche d'une situation individuelle et personnalisée. Devenir coach leur permet de rester dans le champ du conseil tout en ayant une activité plus souple et plus de latitude dans leur organisation temporelle, à l'instar des consultants «professionnels autonomes» (REYNAUD, 2007).

Se présenter comme coach permet enfin d'échapper aux stéréotypes dévalorisants dont pâtit, au sein des entreprises, le titre de psychologue. Le coaching peut ainsi être envisagé comme une manière d'exercer une pratique clinique sans pâtir d'une activité faiblement rémunérée et dévalorisée. Si certaines consultantes le considèrent comme une opportunité pour débuter une activité de psychothérapeute en libéral, elles n'abandonnent pas leur activité de coach, pour des raisons lucratives et afin de se distinguer dans le champ hautement concurrentiel de la psychothérapie. Le coaching sert alors de transition haut de gamme entre le conseil en ressources humaines et la psychothérapie.

\section{Devenir coach, une voie de salut pour des cadres en reconversion professionnelle}

«Je viens du business, donc je suis devenu coach par passion », affirme un ancien cadre commercial. Les cadres en entreprise devenus coachs, qui relèvent $\mathrm{du}$ deuxième grand profil que nous qualifions de «(re) converti», laissent toujours entendre qu'ils ont choisi leur réorientation pour suivre l'appel du cœur et leur vocation, entendue dans sa forme moderne de recherche de «l'authenticité à soi» (BÉRAUD, 2006).
Ils disent avoir renoncé au monde de la performance et de l'argent pour se tourner vers une activité plus enrichissante d'un point de vue personnel, dans une économie des valeurs entre matériel et spirituel. Ce type de récit esthétisé met en scène des reconversions professionnelles importantes, puisque la plupart des cadres reconvertis dans le coaching ont quitté leur entreprise et le salariat, et exercent de toutes autres fonctions que celles qui étaient les leurs pendant la première partie de leur carrière. Ils peuvent, plus rarement, devenir coachs salariés au sein de leur ancienne entreprise, mais toujours au prix d'une rupture avec leur identité professionnelle antérieure. Étudier les spécificités du coaching par rapport à d'autres perspectives professionnelles permet de dégager les conditions qui favorisent la sensibilité des cadres aux discours du «nouvel esprit du capitalisme» et leur engagement dans une de ses activités emblématiques.

Le parcours de Danièle (voir encadré 5) illustre quatre éléments importants communs aux anciens cadres : l'ambivalence entre des ordres de valeur opposés (matériel et existentiel), synthétisés par le coaching; l'impact d'une rupture biographique forte et le rôle joué objectivement et subjectivement par le corps dans la reconversion; l'effet propre des dispositifs comme le bilan de compétences dans l'orientation vers la psychologie; enfin, l'avantage concurrentiel du coaching par rapport à la psychologie universitaire dans la mesure où, à condition d'avoir des ressources économiques, il permet d'accéder à l'emploi sans devoir suivre des études universitaires longues. Nous analysons maintenant plus en détail les différents traits caractéristiques de ce deuxième grand profil des «(re) convertis».

\section{En amont, la rupture professionnelle}

Le projet de devenir coach apparait comme une porte de sortie au terme d'une série d'insatisfactions professionnelles: rupture de la trajectoire professionnelle (licenciement ou démission) parfois corrélée à une rupture d'ordre personnel (accident, divorce, etc.); épreuves de travail, essoufflement des perspectives de carrière. Ces événements conduisent progressivement des cadres plus vulnérables au chômage et moins employables ${ }^{(9)}$ à envisager de se reconvertir professionnellement, majoritairement hors du salariat.

\section{Les effets de rupture durables du licenciement}

La plupart des cadres devenus coachs ont subi un licenciement, du fait notamment des restructurations organisationnelles qui ont eu lieu au sein de certains grands groupes dans les années 1990 et en raison de l'augmentation du chômage des cadres entre 1991 et 1995 puis entre 2001 et 2004 (Pochic, 2009). La

(9) L'employabilité désigne la probabilité de retrouver un emploi. 


\section{Encadré 5 \\ Danièle ou le chemin de Damas (1)}

Fille d'ingénieurs, Danièle commence des études universitaires d'histoire de l'art dans les années 1980. Elle dit avoir ensuite cédé à la pression de son entourage en s'inscrivant en MBA(2) de finance aux États-Unis. Commence pour elle, au tournant des années 1980-1990, une carrière de cadre expatriée dans la finance internationale.

Une insatisfaction croissante, confirmée par la psychanalyse. Tout en détaillant ses avantages matériels de l'époque, elle affirme que cette existence ne lui convenait pas, parlant d'une "carrière comète et [d'une] vie personnelle trou noir" qui la poussent à commencer une psychanalyse. Elle pense s'être alors beaucoup interrogée sur le sens de son travail dans la finance. L'ambivalence imprègne son récit rétrospectif, entre valorisation d'un univers professionnel qualifié et prestigieux, et critique acerbe des valeurs matérielles et de la course à la performance qui le fondent :

"Ce sont des têtes, des tronches, et personne là-dedans n'a la moindre idée du fait que les relations humaines sont plus importantes que de gagner du fric, une grosse voiture ou travailler dix-huit heures par jour. Moi, la première. »

La grande rupture et les «leçons» du corps. À la fin des années 1990, plusieurs événements tragiques précipitent la fin de la carrière financière de Danièle : une rupture avec son compagnon; un grave accident lors de la pratique d'un sport à risque, qui la maintient alitée plusieurs mois; enfin, son licenciement. Elle attaque la banque en justice et gagne le procès, mais la conjonction de ces événements provoque une cassure forte dans sa trajectoire professionnelle. Danièle l'interprète comme un signe : il lui faut changer de vie. Dans une interprétation entre psychosomatique et ésotérisme, elle considère que cette cassure était salutaire et que les événements qu'elle a traversés ne sont pas survenus par hasard :

«Je ne peux pas croire que je n'ai pas appelé une partie de ces symptômes pour me débarrasser de ce truc,

dont je ne savais pas comment me sortir. [...] Et en fait, maintenant, a posteriori, je pense que ça aurait pu être moins grave, mais c'est la meilleure chose qui me soit arrivée dans ma vie. "

Danièle vit sa rupture biographique dans sa chair, ce qui renforce chez elle le sentiment de son individualité(3). Son interprétation en termes de salut - Danièle est sauvée des conséquences de son accident et, selon elle, par ces dernières -, la rend réceptive à une reconversion dans un domaine qu'elle qualifie d' " humain ", reprenant une qualification largement répandue parmi les coachs (voir encadré 1 et l'idée d'humanisme économique).

La réorientation vers la psychologie et la révélation du coaching en entreprise. Au chômage, bénéficiant d'indemnités de licenciement, Danièle fait un bilan de compétences qui débouche sur le projet de devenir psychothérapeute :

«[Du] bilan de compétences, il sort quelque chose, qui est que ce sont plutôt les humains qui m'intéressent, que les yaourts ou les colonnes de chiffres. "

Elle s'inscrit en DESS de psychologie du travail pour obtenir le titre de psychologue mais l'interrompt pour suivre une formation au coaching aux États-Unis, dont elle a entendu parler par son ancien réseau professionnel. Elle se sent en effet en décalage dans la formation universitaire, en raison de son âge (elle a entre 35 et 40 ans) et de son cursus qui, bien que prestigieux, n'a rien à voir avec la psychologie. Danièle retourne le stigmate et critique la formation universitaire, jugeant ses camarades trop jeunes, dénués d'expérience et de réflexivité (elle considère sa psychanalyse comme une formation à part entière) et l'équipe enseignante autoritaire et fermée au dialogue.

À l'inverse, ce qu'elle découvre du coaching aux États-Unis la séduit immédiatement. À Esalen en Californie, berceau du développement personnel, elle suit une formation brève, tournée vers l'action et la réalisation à court terme de ses rêves: "Qu'est-ce que tu vas faire ? Quand est-ce que tu vas le faire ? Où tu vas le faire ?" Et à peine "Est-ce que t'as des freins ?" "Accéder à un métier en contournant des diplômes contraignants explique en grande partie l'attrait qu'elle ressent pour le coaching. Elle commence rapidement à exercer le coaching avec des consultants de son ancien réseau professionnel et prend, quelque temps plus tard, des responsabilités dans une association professionnelle de coachs en France.

(1) En référence à l'épisode biblique de la conversion de Paul de Tarse au christianisme. Sur la route pour Damas, Paul, persécuteur des chrétiens, eut une révélation qui provoqua sa conversion au christianisme et fit de lui un prêcheur convaincu.

(2) Master of business administration.

(3) Nous nous livrons ici à une extrapolation du propos d’Émile DurkнEIM, dans Les formes élémentaires de la vie religieuse (1912) : «Il faut un facteur d'individuation et c'est le corps qui joue ce rôle.» DuRKHEIM montrait que la conscience collective s'incarnait dans les corps et que, ce faisant, elle s'individualisait. En élargissant l'interprétation, on peut considérer que ce qui advient au corps accroît la croyance en l'individualité, et le sentiment de rupture entre «soi» et la société (Elias, 1991). 
séquence - carrière relativement linéaire et ascendante dans une grande entreprise, interrompue par une réorganisation et un licenciement, suivie d'une ou plusieurs tentatives pour retrouver une position similaire dans une autre société et débouchant sur une nouvelle rupture - est répandue. Peu à peu conduits vers le conseil ou la formation dans le secteur professionnel dans lequel ils travaillent jusqu'alors, ils exercent d'abord comme salariés, puis assez vite comme indépendants, leur nouvel emploi ne parvenant pas à déboucher sur une carrière salariée stable. Au-delà des effets de rupture, le licenciement agit comme un avertissement et les amène à réfléchir à la suite de leur carrière (Bouffartigue, 2001). Paradoxalement, la peur du déclassement (CHAUvel, 2006; MAURIN, 2009) les pousse à envisager la sortie d'un système qui ne tient pas ses promesses. Même s'ils retrouvent un poste dans leur secteur d'origine, la menace concrète que représente le licenciement les prédispose à des reconversions comme indépendant, qui ne seraient nullement envisagées si leur carrière initiale avait suivi son cours.

\section{L’insatisfaction professionnelle}

De façon moins brutale que le licenciement, l'essoufflement des perspectives professionnelles ou le plafonnement de leur carrière oriente certains cadres vers le coaching, mais moins systématiquement vers l'indépendance. Leur évolution professionnelle empêchée, des contraintes de travail accrues, ou des changements de hiérarchie ont des effets négatifs, plus importants sur la carrière des femmes cadres, comme l'illustre l'exemple de Lise, une coach interne ${ }^{(10)}$. Ingénieure, docteure en informatique, elle intègre la section recherche et développement d'une grande entreprise industrielle au milieu des années 1980. Alors que des responsabilités hiérarchiques qui l'éloignent de son domaine de spécialité lui sont confiées, elle commence à se sentir dépassée sur les questions techniques et à chercher d'autres sources de légitimité dans la communication. Si le dilemme entre expertise et responsabilité managériale est classique (Роснic, 2004), l'éloignement de la «technique» pour se rapprocher de «l'humain» est un discours répandu chez les cadres ingénieures, plus disposées à le tenir que leurs homologues masculins, et davantage poussées à s'orienter vers des fonctions de service (Stevens, 2007). Le décalage s'aggrave lorsque Lise est promue à une fonction de direction qui implique une mobilité géographique et davantage de temps de présence au travail, tandis qu'elle traverse une crise familiale liée à son divorce. L'investissement exigé par son ascension professionnelle entre en contradiction avec ses contraintes familiales accrues (elle a trois enfants); les deux deviennent incompatibles et la conduisent à quitter son nouveau poste, ce qui compromet sa

(10) Entretiens réalisés le 12 février, puis le 10 mars 2008. carrière. C'est dans ce contexte qu'elle découvre le coaching, via une formation managériale initiée par son entreprise. Le parcours de Lise est typique d'une auto-exclusion de la voie des grands dirigeants, dont les règles de «fabrication organisationnelle» sont tracées au masculin (Guillaume, Pochic, 2007). Elle parvient à se reconvertir comme coach interne dans son entreprise dans le cadre de la mise en place de politiques néomanagériales et reste ainsi salariée et cadre. Elle souligne qu'elle aurait eu des difficultés à s'établir comme coach indépendante et qu'elle ne sait pas si elle serait devenue coach si l'entreprise n'avait pas soutenu son projet.

\section{Des cadres plus vulnérables et moins employables?}

Les cadres qui se sont réorientés vers le coaching représentent des catégories à la fois plus vulnérables au chômage et moins employables que l'ensemble des cadres des grandes entreprises. S'ils sont sensibles aux discours sur les «carrières nomades» (ARTHUR, RousseAu, 1996), ils représentent en réalité la part minoritaire des cadres concernés par les décrochages de carrière et le «nomadisme» qui en découle. Ce sont en effet d'abord les cadres commerciaux, qui sont les plus vulnérables au chômage, avec les plus jeunes et les moins diplômés (Pochic, 2009), et ceux qui travaillent dans les secteurs de l'informatique et de la finance, particulièrement affectés par les fluctuations de l'activité économique, qui se réorientent vers le coaching. On trouve également parmi eux des cadres moins employables en raison de leur âge (plus de 45 ans). Les coachs internes, en général diplômés d'une grande école d'ingénieurs ou de commerce, illustrent a contrario la plus forte vulnérabilité au chômage des cadres reconvertis comme coachs externes : les plus diplômés restent les plus protégés sur le plan de l'emploi.

Néanmoins, mêmes victimes d'un licenciement et du chômage, les cadres demeurent protégés par leur statut, bénéficiant de dispositifs individualisés d'aide au reclassement, ce qui leur permet de débuter comme indépendant dans des conditions économiques et subjectives favorables. Un ancien directeur commercial, licencié à plus de 50 ans lors d'une restructuration au début des années 2000, après vingt ans passés au sein de son entreprise, voit son licenciement «comme une opportunité» en raison de son «épuisement professionnel». Il souligne qu'il part dans «de très bonnes conditions matérielles» et qu'il «bénéficie d'un accompagnement dans le cadre d'un outplacement ${ }^{(11) » .}$

(11) Littéralement «reclassement externe» d'un salarié, l'outplacement désigne l'accompagnement individuel d'un cadre licencié dans le but de l'aider à retrouver un emploi. 


\section{Le rôle d'orientation des dispositifs d'accompagnement psychologique}

La plupart des cadres devenus coachs ont suivi une psychothérapie ou une psychanalyse, souvent avant leur reconversion. D'autant plus forte qu'elle survient dans un contexte personnel éprouvant, la rencontre avec la psychothérapie semble, aux dires des cadres, les avoir sensibilisés à un ordre de valeurs qu'ils qualifient d' "éthique» et de "philosophie de vie » et qu'ils présentent comme une alternative à celui du monde économique. Tout en étant susceptibles de les avoir rendus plus enclins à quitter des emplois qui ne leur convenaient plus, leur nouveau cadre de valeurs leur a également offert des perspectives professionnelles pour mieux «rebondir» après un licenciement. Plusieurs l'évoquent pour justifier l'ouverture du champ des possibles professionnels aux métiers de «l'accompagnement », dont le coaching est l'une des concrétisations envisageables. La psychothérapie est alors convoquée pour justifier leur évolution de l'un à l'autre monde.

Toutefois ces dispositions seraient sans doute restées latentes si les cadres concernés n'avaient pas été confrontés à des dispositifs professionnels psychologisants. Conçus pour développer la réflexivité sur les questions de travail, de carrière ou d'emploi, les formations managériales, le bilan de compétences, l'outplacement et le coaching sont proposés aux cadres par les entreprises ou des organismes publics. Souvent effectués par des consultants formés à des techniques de psychologie, ils participent à la diffusion d'une culture psychologique auprès d'un public peu familier des sciences humaines, mais particulièrement sensible aux techniques de rationalisation de sa propre vie (GADÉA, 2003). Ils contribuent ainsi à aiguiller ou à renforcer l'orientation de ces cadres vers des fonctions moins opérationnelles, plus relationnelles, proches de la psychologie.

Beaucoup d'anciens cadres disent avoir amorcé leur reconversion à la faveur d'une formation, et souvent même d'un coaching. Dans le champ des possibles ouvert par le coaching, l'option de devenir précisément coach paraît étonnamment prégnante. Les coachs n'orientent probablement pas sciemment leurs coachés vers ce projet ${ }^{(12)}$ mais il semble se mettre en place une forme de circularité et de cooptation informelle entre coachs et coachés, conduisant certains de ces derniers vers cette activité. Devenir coach après avoir été soi-même coaché est une forme de «contre-don» (aider comme on a été aidé) mais correspond aussi, plus pragmatiquement, à une perspective professionnelle dans une

(12) Néanmoins, une partie des coachs tirent une partie de leurs revenus de la formation au coaching et peuvent être incités à recruter de nouveaux stagiaires. Lors de notre observation participante comme coachée, nous avons été confrontée à des pressions de la part de «notre» coach pour nous convaincre de suivre un cycle complet de formation au coaching. situation de blocage, perspective rendue tangible par le contact établi avec le coach.

\section{Les promesses d'émancipation du coaching}

C'est aux mérites propres du coaching, étant entendu qu'ils doivent être rapportés aux autres possibilités de reconversion offertes aux cadres pour la seconde partie de leur carrière (PoussouPlesse, 2007), qu'il faut maintenant s'atteler pour comprendre en quoi il cristallise leurs aspirations.

Si les cadres sont marqués par leur psychothérapie, c'est comme coach qu'ils se reconvertissent plutôt que comme psychothérapeute. En restant dans le champ de l'entreprise, ils peuvent valoriser la première partie de leur carrière professionnelle et réinvestir leurs compétences, leur carnet d'adresses et leur diplôme initial. De plus, la promotion symbolique est importante, puisqu'ils aident et conseillent ceux qu'ils étaient encore hier. Se reconvertir dans le coaching est également une promesse de réorientation à court terme, qui dispense de l'acquisition parfois longue de nouvelles qualifications. Enfin, il offre une porte de sortie professionnelle en promettant un emploi a priori rémunérateur(13) hors des contraintes organisationnelles à des cadres déçus qui se montrent alors séduits par l'argumentaire de l'indépendance.

\section{Un faible " coût " d'entrée}

L'activité de coach n'est pas réglementée et, comme nous l'avons vu, une formation n'est pas obligatoire, bien qu'elle soit de plus en plus répandue. Les formations au coaching restent brèves, même si leur durée s'est allongée pour atteindre une vingtaine de jours en moyenne (sept ou huit weekends de deux ou trois jours, étalés sur une année). Elles ne sont pas sanctionnées par un diplôme national qui restreindrait l'accès à la profession. La principale barrière à l'entrée est économique car les formations coûtent cher (4000 euros pour les formations universitaires, plus de 10000 euros pour la plus prestigieuse), mais elles restent abordables pour d'anciens cadres supérieurs, même chômeurs (dans la période d'indemnisation) et pour une grande partie des consultants, d'autant plus qu'elles sont parfois financées par des dispositifs d'aide au retour à l'emploi. Suivre une formation au coaching est plus accessible et rapide que reprendre des études universitaires, de psychologie par exemple, qui demandent un niveau de diplôme minimal, un investissement de plusieurs années et nécessitent la sanction de multiples examens.
(13) Le tarif horaire d'un coaching individuel varie entre 250 et 450 euros dans les grandes entreprises. Un contrat de coaching se vend entre 5000 et 10000 euros. 


\section{Les "choix" de l'indépendance}

L'exercice du coaching se fait la plupart du temps hors du salariat, «en libéral», ce que les coachs revendiquent comme le signe de leur indépendance à l'égard des organisations. Le désir de devenir coach rencontre fréquemment celui de se mettre à son compte, lui offrant un support concret. Ce discours est plutôt tenu par des hommes, tel cet ancien directeur commercial, Pascal, qui retrouve rapidement un travail après son licenciement à 50 ans, mais dans des fonctions éloignées de ses expériences antérieures : son nouvel emploi de directeur d'un syndicat «se passe très mal» et représente «le deuil de [sa] situation professionnelle passée», le faisant "[tomber] en dépression». "Je fais [alors] le choix de l'autonomie et de l'indépendance, et je m'intéresse au coaching ", affirme-t-il(14). Le statut d'indépendant matérialise la rupture avec sa situation professionnelle passée et n'autorise plus (ou autorise moins) de comparaison avec son statut antérieur : en effet, celle-ci aurait été au désavantage de sa situation présente, étant donné les perspectives d'emploi des «seniors» en France. Changement majeur, ce statut d'indépendant est la promesse d'un nouveau départ dans un nouveau contexte. Thierry, 45 ans, ancien ingénieur devenu consultant suite à un licenciement, va encore plus loin en affirmant à quel point l'objectif de créer sa propre entreprise primait sur celui de devenir coach : "Mon objectif, c'était pas d'être coach, c'était de fonder une compagnie ${ }^{(15)}$.»

Ce qui est présenté comme un «choix» de l'indépendance comporte néanmoins des risques et n'est pas sans poser des difficultés, notamment économiques. C'est ce que soulignent certaines femmes : Corinne, ancienne cadre commerciale, pourtant propriétaire de son lieu d'habitation, touchant des indemnités de licenciement, dotée d'économies personnelles, et dont le conjoint travaille, présente ainsi son nouveau statut de coach à son compte comme une prise de risque et le «renoncement à certaines habitudes de confort matériel». L'indépendance est alors moins présentée comme un objectif en soi que comme une condition nécessaire pour devenir coach, bien que la valorisation de l'indépendance reste forte dans le monde social du coaching (voir encadré 1 et la valorisation de l'«entreprise de soi»). C'est ce qu'expriment plusieurs femmes cadres qui ont dû quitter les cabinets de conseil dans lesquels elles étaient entrées après leur licenciement, au motif que «devenir coach, ce n'était pas le métier de la boutique». Le cas des coachs internes illustre en creux les limites du «choix» de l'indépendance: les cadres supérieurs des grandes entreprises qui n'ont pas été licenciés cherchent d'abord à rester salariés plutôt

(14) Entretien réalisé le 2 mars 2007

(15) Entretien réalisé le 22 mai 2007. qu'à devenir indépendants, surtout quand ils ont charge de famille.

Le passage à l'indépendance est un choix contraint pour ces cadres, qui fait suite à des accidents de parcours et à leur difficile réversibilité. Même si leur situation, dans l'ensemble, n'est pas aussi fragile que celle de certains cadres au chômage, pour qui le fait de devenir entrepreneur ou de se mettre à son compte est «parfois la seule manière de retrouver un emploi» (Pochic, 2009, p. 388), il reste que le tournant de l'indépendance est parfois la seule solution pour éviter un décrochage trop patent de leur carrière et tenter un nouveau départ. Pour ces cadres déçus par des organisations qui les ont licenciés ou qui n'ont pas satisfait leurs attentes de carrière, le coaching représente une perspective concrète de se mettre à leur compte. L'indépendance se confond avec le projet de devenir coach et offre une voie de salut professionnel qui leur permet d'enchanter leur parcours.

$$
\begin{gathered}
* \\
* *
\end{gathered}
$$

Prendre au sérieux la diversité des engagements dans le coaching permet d'enrichir la compréhension des voies par lesquelles se diffuse le «nouvel esprit du capitalisme», en montrant les intérêts et les attentes à partir desquels un ensemble d'acteurs s'approprie ses représentations typiques. L'invention d'un groupe professionnel comme celui des coachs satisfait les intérêts différenciés mais convergents de deux principaux types de population : des consultants qui améliorent leur position économique et sociale et des cadres qui se «recyclent» durant la deuxième partie de leur carrière.

Des consultants et formateurs à la trajectoire professionnelle buissonnante et irrégulière, peu diplômés ou dotés de diplômes ne correspondant pas à l'espace professionnel embrassé, trouvent ainsi dans le coaching une opportunité; ils valorisent du coup leur parcours d' «entrepreneurs d'eux-mêmes », l'importance des «rencontres» et des relations au détriment des qualifications, et masquent les conditions sociales qui les ont conduits au coaching. Le coaching leur permet aussi de contourner la rareté des débouchés dans le champ de la santé mentale et de quitter les cabinets de conseil pour ceux qui, parmi eux, ont été formés à la psychologie. Ce sousprofil illustre une tendance interne au conseil, qui lie l'affirmation d'une nouvelle orientation et d'une nouvelle pratique à des revendications en termes de statut d'emploi. Ces consultants mêlent ainsi la valorisation de la flexibilité comme émancipation $\mathrm{du}$ salariat à celle de l'humanisation des entreprises, renouant avec l'une des utopies originelles du conseil (Henrr, 2009). Le coaching a enfin constitué une voie de salut pour des cadres d'entreprise qui ont vécu une rupture dans leur trajectoire 
relativement linéaire. Ces anciens cadres se font les chantres de la revendication de l'indépendance et de la critique des organisations, de l'entreprise de soi, mais aussi de l'humanisation des entreprises, certains restant d'ailleurs des coachs internes salariés comme nous l'avons vu.

Ces différentes trajectoires s'inscrivent dans des configurations sociohistoriques spécifiques. Elles dépendent des transformations du travail et de l'emploi des trente dernières années. En particulier, la multiplication des statuts «hybrides» d'emploi et le renouveau du travail indépendant (BERNARD, Dressen, 2014) jouent un rôle majeur dans l'essor des vocations pour le coaching, devenu attractif aussi bien pour d'anciens cadres qui ont connu une rupture avec le salariat et un passage par le chômage, que pour des acteurs à la trajectoire professionnelle irrégulière, voire durablement écartés du marché du travail, tels les consultants au parcours éclectique. Si les coachs se font les vecteurs du «nouvel esprit du capitalisme», ils contestent un système qui a peiné à leur faire de la place (pour les consultants) ou les a rejetés après les avoir acceptés (pour les cadres [re]convertis). Les revendications libérales, responsabilisantes et individualisantes des coachs reflètent ainsi leurs difficultés à trouver ou à garder une place dans un système où le recrutement et l'avancement de carrière sont largement fondés sur les qualifications et les classements - hiérarchie des diplômes, suprématie des grandes écoles, elles-mêmes hiérarchisées dans le monde des cadres (Cousin, 2008). Conduits à exercer une activité qui leur offre une promotion symbolique et, pour certains, également économique, ils sont enclins à tenir dans les entreprises des discours typiques de ce «nouvel esprit» et de ses critiques de la planification, des plans de carrière, et de la hiérarchie, dont ils ont eux-mêmes expérimenté les limites. Ils rappellent les «entrepreneurs» et les «dépossédés» des sociétés traditionnelles décrits par Georges BALANDIER (1974), qui ont tout à gagner à une innovation.

\section{Bibliographie}

Аввотт A. D. (1988), The system of professions: an essay on the division of expert labor, Chicago, University of Chicago Press.

Arthur M. B., Rousseau D. M. (ed.) (1996), The boundaryless career: a new employment principle for a new organizational era, New York, Oxford University Press.

Balandier G. (1974), Anthropo-logiques, Paris, Presses universitaires de France.

Barley S. R., Kunda G. (2004), Gurus, hired guns, and warm bodies: itinerant experts in a knowledge economy, Princeton, N. J., Princeton University Press.

BÉRAUD C. (2006), Le métier de prêtre. Approche sociologique, Paris, Éditions de l'Atelier.

Bernard S., Dressen M. (2014), «Penser la porosité des statuts d'emploi», La nouvelle revue du travail [en ligne], $\mathrm{n}^{\circ} 5$, consulté le 7 janvier 2016

Berrebi-Hoffmann I. (2002), "Les multinationales de l'expertise», Sociologies pratiques, ${ }^{\circ}$ 6, pp. 47-69.

Boltanski L. (1981), «America, America... Le plan Marshall et l'importation du "management"», Actes de la recherche en sciences sociales, $\mathrm{n}^{\circ} 38, \mathrm{pp} .19-41$.

Boltanski L., Chiapello È. (1999), Le nouvel esprit du capitalisme, Paris, Gallimard.

Bouffartigue P. (2001), Les cadres. Fin d'une figure sociale, Paris, La Dispute.

Bourdieu P. (1979), La distinction : critique sociale $d u$ jugement, Paris, Éditions de Minuit.
Champion F. (dir.) (2008), Psychothérapie et société, Paris, Armand Colin.

Chauvel L. (2006), Les classes moyennes à la dérive, Paris, Seuil.

Chiapello È. (1998), Artistes versus managers: le management culturel face à la critique artiste, Paris, Éditions Métailié.

Cousin O. (2008), Les cadres à l'épreuve du travail, Rennes, Presses universitaires de Rennes.

Crozier M., Friedberg E. [1977] (1992), L'acteur et le système: les contraintes de l'action collective, Paris, Seuil.

Denave S. (2015), Reconstruire sa vie professionnelle. Sociologie des bifurcations biographiques, Paris, Presses universitaires de France.

Detienne M., Vernant J.-P. (1974), Les ruses de l'intelligence : la «mètis » des Grecs, Paris, Flammarion.

DuRKHeIM É. [1912] (2008), Les formes élémentaires de la vie religieuse: le système totémique en Australie, Paris, F. Alcan.

Elias N. (1991), La société des individus, Paris, Fayard.

GADÉA C. (2003), Les cadres en France : une énigme sociologique, Paris, Belin.

Giraud B. (2007), «Le syndicalisme saisi par le management. Les conditions de production d'une expertise managériale de l'action syndicale au prisme de ses investissements diversifiés », Politix, $\mathrm{n}^{\circ} 79$, pp. 125-147. 
Guillaume C., Pochic S. (2007), «La fabrication organisationnelle des dirigeants. Un regard sur le plafond de verre», Travail, genre et sociétés, $\mathrm{n}^{\circ}$ 17, pp. 79-103.

Henry O. (1992), «Entre savoir et pouvoir. Les professionnels de l'expertise et du conseil», Actes de la recherche en sciences sociales, $\mathrm{n}^{\circ} 95, \mathrm{pp} .37-54$.

Henry O. (2009), «Organisation rationnelle de l'humanité et "humanisation" du gouvernement des entreprises », in Berrebi-Hoffmann I. (dir.), Politiques de l'intime. Des utopies sociales d'hier aux mondes du travail d'aujourd'hui, Paris, La Découverte, pp. 57-71.

Henry O. (2012), Les guérisseurs de l'économie. Ingénieurs-conseil en quête de pouvoir. Sociogenèse du métier de consultant (1900-1944), Paris, CNRS éditions.

KipPING M., ENGWALl L. (2002), Management consulting: emergence and dynamics of a knowledge industry, New York, Oxford University Press.

LARQuier DE G., RÉMILlon D. (2008), «Assiste-t-on à une transformation uniforme des carrières professionnelles vers plus de mobilité ? Une exploitation de l'enquête Histoire de vie», Travail et emploi, $\mathrm{n}^{\circ} 113$, pp. 13-30.

LE Goff J.-P. (1999), La barbarie douce: la modernisation aveugle des entreprises et de l'école, Paris, La Découverte.

MAURIN É. (2009), La peur du déclassement. Une sociologie des récessions, Paris, Seuil.

Menger P.-M. (2002), Portrait de l'artiste en travailleur. Métamorphoses du capitalisme, Paris, Seuil.

NÉGRONI C. (2007), Reconversion professionnelle volontaire. Changer d'emploi, changer de vie. Un regard sociologique sur les bifurcations, Paris, Armand Colin.

PinTo V. (2010), «Une pédagogie anti-scolaire aux frontières de l'école. Le cas des formateurs BAFA», in de Lescure E., Frétigné C. (dir.), Les métiers de la formation. Approches sociologiques, Rennes, Presses universitaires de Rennes, pp. 197-207.

Pochic S. (2004), «Le manager et l'expert: des figures imposées ?», in Karvar A., Rouban L. (dir.), Les cadres au travail. Les nouvelles règles du jeu, Paris, La Découverte, pp. 168-198.

Pochic S. (2009), «Les cadres et la menace du chômage : mobilisation catégorielle contre un risque récurrent», in Demazière D., Gadéa C. (dir.), Sociologie des groupes professionnels : acquis récents et nouveaux défis, Paris, La Découverte, pp. 378-390.

Poussou-Plesse M. (2007), «Du principe d'une "seconde partie de carrière" à la défense d'une cause des "quinquas". Étude de cadres seniors en "repositionnement professionnel"», in Reguer D. (dir.), Vieillissement et parcours de fins de carrières : contraintes et stratégies, Ramonville Saint-Agne, Éditions Érès, pp. 125-156.
Prost A. (2008), « Jalons pour une histoire de la formation des adultes (1920-1980)», in Laot F., de Lescure E. (dir.), Pour une histoire de la formation, Paris, L'Harmattan, pp. 37-54.

REYNAUd E. (2007), «Aux marges du salariat, les professionnels autonomes», in Vatin F. (dir.), Le salariat. Théorie, histoire et formes, Paris, La Dispute, pp. 299-310.

Salman S. (2003), «La carrière d'un “fondateur” du coaching», Terrains et travaux, $\mathrm{n}^{\circ} 4$, pp. 140-161.

SALMAN S. (2008), «La fonction palliative du coaching en entreprise», Sociologies pratiques, ${ }^{\circ} 17$, pp. 43-54.

Salman S. (2013), Une hygiène psychique au travail ? Genèse et usages du coaching en entreprise en France, Thèse de doctorat de sociologie, Université Paris-Ouest Nanterre-La Défense.

SALman S. (2014), «Un coach pour battre la mesure ? La rationalisation des temporalités de travail des managers par la discipline de soi», Revue d'anthropologie des connaissances, vol. 8, $\mathrm{n}^{\circ}$ 1, pp. 97-122.

SEnTin J.-L. (2010), «Nous sommes tous des marginaux sécants », in Devienne É. (dir.), Le grand livre de la supervision, Paris, Eyrolles, pp. 127-142.

Simmonet V., Ulrich V. (2009), «La mobilité entre métiers : 30\% des emplois en 1998 avaient changé de métier en $2003 »$, Premières informations premières synthèses, $\mathrm{n}^{\circ}$ 05-3, Paris, Dares.

SOCIÉTÉS CONTEMPORAINES (2013), «Les entrepreneurs de la "nouvelle entreprise"», Sociétés contemporaines, $\mathrm{n}^{\circ} 89$.

Stevens H. (2007), «Destins professionnels des femmes ingénieures. Des retournements inattendus», Sociologie du travail, vol. 49, $\mathrm{n}^{\circ}$ 4, pp. 443-463.

Stevens H. (2011), «De l'intervention psychosociologique au "développement personnel" dans l'entreprise. Esquisse d'une généalogie des relations entre management et psychologie en France», Regards sociologiques, $\mathrm{n}^{\circ}$ 41-42, pp. 57-74.

SteVens H. (2012), «Autonomie récusée, autonomie fabriquée. Informaticiens à l'épreuve de l'Entreprise de soi», Genèses, vol. 2, n 87, pp. 90-112.

TANGuY L. (2001), «Les promoteurs de la formation en entreprise (1945-1971)», Travail et emploi, $\mathrm{n}^{\circ} 86$, pp. 27-47.

Thine S., Lagneau-Ymonet P., Denord F., Caveng R. (2013), «Entreprendre et dominer. Le cas des consultants », Sociétés contemporaines, $\mathrm{n}^{\circ}$ 89, pp. 73-99.

Villette M. (2003), Sociologie du conseil en management, Paris, La Découverte. 